\title{
THE S-MATRIX FOR SYSTEMS WITH BOUND STATES
}

\author{
by TH. W. RUIJGROK
}

Instituut voor theoretische fysica der Rijksuniversiteit, Utrecht, Nederland.

\section{Synopsis}

A unitary $S$-matrix is defined for a system of three particles, two of which can form a bound state. It is shown how for elastic scattering the polarization of the bound state must be taken into account.

$\S 1$. Introduction. The problem of scattering by a composite particle has an extra complication when compared with other three or more particle problems. In addition to the general difficulties of solving the Schrödinger equation there is the fact that for systems with bound states no practically useful answer has been given to the following two questions:

$\propto)$ how should the $S$-matrix be defined?

$\beta$ ) how should the $S$-matrix be calculated in a systematic way?

In order to locate the difficulties we first show how for a system without bound states the $S$-matrix can be defined. For convenience we consider a system which can be described by a Schö̈dinger equation with Hamiltonian $H=H_{0}+V$. Let the states $|\mu\rangle$ be a complete set of orthonormal eigenstates of $H_{0}$ with energies $\varepsilon_{\mu}$. In the case without bound states there is a one-to-one correspondence between these states and the eigenstates of $H$. Three different sets of cigenstates are distinguished:

$$
\begin{aligned}
& |\mu\rangle_{ \pm}=|\mu\rangle-\int_{v} \frac{C_{\nu \mu}( \pm)}{\left(\varepsilon_{v}-\varepsilon_{\mu} \mp i 0\right)}|v\rangle \\
& |\mu\rangle_{0}=N(\mu)|\mu\rangle-\int_{v} \frac{C_{\nu \mu}(0)}{\left(\varepsilon_{v}-\varepsilon_{\mu}\right)_{P}}|\nu\rangle
\end{aligned}
$$

$N(\mu)$ has to be chosen such that the states $|\mu\rangle_{0}$ are normalized. Consider now an arbitrary solution of the Schrödinger equation

$$
|\varphi(t)\rangle=\int_{\mu} d_{+}(\mu) \mathrm{e}^{-i \varepsilon_{\mu} t}|\mu\rangle_{+}=\int_{\mu} d_{-}(\mu) \mathrm{e}^{-i \varepsilon_{\mu} t}|\mu\rangle_{-}
$$

For large times this state can be expanded in the unperturbed states:

and

$$
|\varphi(t)\rangle \simeq \int_{\mu} \tilde{b}(\mu) \mathrm{e}^{-i \varepsilon_{\mu} t}|\mu\rangle \text { for } t \rightarrow+\infty
$$

$$
|\varphi(t)\rangle \simeq \int_{\mu} b(\mu) \mathrm{e}^{-i \varepsilon_{\mu} t}|\mu\rangle \quad \text { for } \quad t \rightarrow-\infty
$$


The $S$-matrix is defined by

$$
\tilde{b}(\mu)=\int_{\nu} S_{\mu \nu} b(v)
$$

An explicit expression for $S_{\mu \nu}$ can be obtained when the expansion $\left(1.1_{+}\right)$is inserted into (1.2) and use is made of the formula

$$
\lim \frac{\mathrm{e}^{i\left(\varepsilon_{\mu}-\varepsilon_{\nu}\right) t}}{\varepsilon_{\mu}-\varepsilon_{\nu}-i 0}=\left\{\begin{array}{cl}
2 \pi i \delta\left(\varepsilon_{\mu}-\varepsilon_{\nu}\right) & \text { for } t \rightarrow+\infty \\
0 & \text { for } t \rightarrow-\infty
\end{array}\right.
$$

We find

$$
S_{\mu \nu}=\delta(\mu-\nu)-2 \pi i C_{\mu \nu}(+) \delta\left(\varepsilon_{\mu}-\varepsilon_{v}\right) .
$$

It can be proved that for this $S$-matrix and the state $|\varphi(t)\rangle$ as given by (1.2) the following relations hold:

$$
\begin{aligned}
& \lim _{t \rightarrow \mp \infty}\left[|\varphi(t)\rangle-\int_{\mu} d_{ \pm}(\mu) \mathrm{e}^{-i \varepsilon_{\mu} t}|\mu\rangle\right] \Rightarrow 0 \\
& \lim _{t \rightarrow+\infty}\left[|\varphi(t)\rangle-\int_{\nu \mu} S_{\mu \nu} d_{+}(\nu) \mathrm{e}^{-i \varepsilon_{\mu} t}|\mu\rangle\right] \Rightarrow 0 \\
& \lim _{t \rightarrow-\infty}\left[|\varphi(t)\rangle-\int_{\nu \mu} S_{\nu \mu}^{*} d_{-}(v) \mathrm{e}^{-i \varepsilon_{\mu} t}|\mu\rangle\right] \Rightarrow 0
\end{aligned}
$$

The double arrow indicates that the norm of the states between brackets approaches zero. A proof can be found in reference ${ }^{1}$ ) p. 347. The only extra information needed is

$C_{\mu \nu}(-)=C_{\nu \mu}^{*}(+)$ for $\varepsilon_{\mu}=\varepsilon_{\nu}$, which is not difficult to prove $\left.{ }^{2}\right)$. An immediate consequence of (1.8) is

$$
S_{\mu \nu}=-\langle\mu \mid v\rangle_{+}
$$

and hence the unitarity of the $S$-matrix. (1.9) is sometimes taken as the definition of $S$. The property (1.8a) is expressed by saying that the states $|\mu\rangle$ are asymptotically stationary (a.s.). For what follows it is important to realize that in the case discussed above there exists a set of a.s. states, which are linearly independent and in a one-to-one correspondence with the exact stationary states. The expansion (1.3) can therefore be made in a unique way and the $S$-matrix is then also determined uniquely. In the case of scattering by a composite particle it is not immediately clear how linearly independent states must be defined. Let us consider a system of three different particles of which only no. 2 and no. 3 can form a bound state. We know that for large times this system behaves either as three free particles, or as two free particles, one of which is composite. (We exclude the possibility of three particles bound together. For scattering problems this state does not play a role anyway). If we now represent these states by products of one-particle plane wave states, it follows from the completeness of the three-particle states that the above mentioned two-particle states cannot be added as independent states without making the system over- 
complete. As a consequence the definition (1.4) of the $S$-matrix is no longer unique. Also the Schrödinger equation, when written in terms of these states, does not have a unique solution. These and other problems are discussed (but not solved) in the review article by Brenig and $\mathrm{Haag}{ }^{3}$ ), where also more references on the subject are given. Also J a u ch's ${ }^{4}$ ) treatment does not solve the scattering problem for many channels. We mention the theory of the $R$-matrix ${ }^{5}$ ) for nuclear reactions, for which the same - and other ${ }^{6}$ ) - difficulties exist as for the $S$-matrix.

A number of attempts ${ }^{7}$ ) has been made to answer question $\beta$ ), but the range of validity of the assumptions that had to be made is not fully understood.

The aim of the present paper is to give a new answer to question $\alpha$ ) on the basis of which question $\beta$ ) may be attacked. The idea is to introduce a.s. states, which will provide a description of all possible states at large times. The a.s.states will differ from the "basic states" defined by Ekstein ${ }^{8}$ ) in that they will be linearly independent. A unitary $S$-matrix is then defined, which follows from the expansion of the stationary states in a.s. states. The Schrödinger equation takes a simple form in this representation. In the case of elastic scattering (e.g. of a proton by a deuteron) the equation reduces to a simple two-particle problem and it is clearly seen how the polarization of the bound state must be taken into account. For convenience of presentation we will discuss only the case of three particles of different kind. No explicit examples are considered, but the method may be applied to elastic scattering like $p-d, n-d, e-H$ or $p-H$.

§2. The a.s. states and the S-matrix. We consider a system of spinless particles of three different kinds, 1, 2 and 3, but with the same mass. The Hamiltonian is $H=H_{0}+V$, where $H_{0}$ is the total kinetic energy and

$$
V=\frac{1}{2} \sum_{i \neq j} v_{i j}\left(\boldsymbol{r}_{i}-\boldsymbol{r}_{j}\right) \quad(i, j=1,2,3) .
$$

A lower index will always refer to the corresponding particle type. We introduce the following notation:

$\left|\boldsymbol{k}_{1}\right\rangle$ is a one-particle eigenstate of $H_{0}$ (and of $H$ ) with momentum $\boldsymbol{k}_{1}$ and energy $\varepsilon\left(k_{1}\right)$;

$|\mu\rangle$ is a two-particle eigenstate of $H_{0}$ of particles of the second and third kind and with the energy $\varepsilon_{\mu}$;

$\left|\boldsymbol{k}_{1} \mu\right\rangle=\left|\boldsymbol{k}_{1}\right\rangle|\mu\rangle$ is a three-particle eigenstate of $H_{0} ;|a\rangle$ is an eigenstate of $H$ corresponding to a bound state of particles 2 and 3 and with energy $\omega_{a}$; we assume that no other pairs of particles can be bound;

$|\mu\rangle_{ \pm}$and $|\mu\rangle_{0}$ are states of particles 2 and 3 , which are eigenstates of $H$ with energy $\varepsilon_{\mu}$; we assume that $\varepsilon_{\mu}$ is the same as the unperturbed energy. The distinction between the three types of scattering states is given in (1.1). 
The states $|a\rangle$ together with any of the sets $|\mu\rangle_{ \pm}$or $|\mu\rangle_{0}$ form a complete and orthonormal basis in the two-particles space.

The a.s. states are now defined as product states in the following way:

$$
\begin{aligned}
& \left|\boldsymbol{k}_{1} a\right\rangle_{a s} \equiv\left|\boldsymbol{k}_{1}\right\rangle|a\rangle \\
& \left|\boldsymbol{k}_{1} \mu\right\rangle_{a s}^{0} \equiv\left|\boldsymbol{k}_{1}\right\rangle|\mu\rangle_{0}
\end{aligned}
$$

In general these states will be written as $|\alpha\rangle_{a s}^{0}$. We will prove that the a.s. states have the following properties

a) they form a complete set of linearly independent states.

b) they are asymptotically orthonormal

c) they are asymptotically stationary.

A.s. states with exactly the same properties were introduced by Van Hove $\left.{ }^{1}\right)$ to describe persistent perturbation effects, like those occurring in field theory.

a) This follows immediately from the facts that in the two-particle space the states $|\mu\rangle$ form a complete set and that all the states $|a\rangle$ and $|\mu\rangle_{0}$ together are linearly independent.

b) In the present case it is seen immediately that ${ }_{a s}^{0}\langle\alpha \mid \beta\rangle_{a s}^{0}=\delta(\alpha-\beta)$. When two or three particles are indistinguishable the $|\alpha\rangle_{a s}^{0}$ have to be defined in such a way that

1) they are properly symmetrized

2) they are asymptotically orthonormal, i.e. if

$$
\begin{gathered}
\left|\varphi^{(i)}(t)\right\rangle=\int_{\alpha} d(i, \alpha) \mathrm{e}^{-i \epsilon_{\alpha} t}|\alpha\rangle_{a s}^{0} \text { then } \\
\lim _{t \rightarrow \pm \infty}\left\langle\varphi^{(1)}(t) \mid \varphi^{(2)}(t)\right\rangle=\iint_{\alpha} d^{*}(1, \alpha) d(2, \alpha)
\end{gathered}
$$

$\varepsilon_{\alpha}$ is defined as follows:

$$
\begin{aligned}
& \varepsilon_{\alpha}=\varepsilon\left(k_{1}\right)+\omega_{a} \text { for the state } \\
& \varepsilon_{\alpha}=\varepsilon\left(k_{1}\right)+\varepsilon_{\mu} \text { for the state }
\end{aligned}
$$

c) Since the $|\alpha\rangle_{a s}^{0}$ are linearly independent they can be used to characterize a set of eigenstates of $H$, which is not overcomplete. This is done by constructing eigenstates with energy $\varepsilon_{\alpha}$, which are of the form

$$
|\alpha\rangle_{0 \pm}=|\alpha\rangle_{a s}^{0}-\int_{\beta} \frac{C_{\beta \alpha}( \pm)}{\varepsilon_{\beta}-\varepsilon_{\alpha} \mp i 0}|\beta\rangle_{a s}^{0}
$$

Defining an $\mathscr{S}$-matrix by

$$
\mathscr{S}_{\alpha \beta}=\delta(\alpha-\beta)-2 \pi i C_{\alpha \beta} \delta\left(\varepsilon_{\alpha}-\varepsilon_{\beta}\right),
$$

it can be shown that, with

$$
|\varphi(t)\rangle=\int_{\alpha} d_{+}(\alpha) \mathrm{e}^{-i \varepsilon_{\alpha} t}|\alpha\rangle_{0+}=\int_{\alpha} d_{-}(\alpha) \mathrm{e}^{-i \varepsilon_{\alpha} t}|\alpha\rangle_{0-},
$$


the following relations hold:

$$
\begin{aligned}
& \lim _{t \rightarrow+\infty}\left[|\varphi(t)\rangle-\int_{\alpha} d_{ \pm}(\alpha) \mathrm{e}^{-i \varepsilon_{\alpha} t}|\alpha\rangle_{a s}^{0}\right] \Rightarrow 0 \\
& \lim _{t \rightarrow+\infty}\left[|\varphi(t)\rangle-\int_{\alpha \beta} \mathscr{S}_{\alpha \beta} d_{+}(\beta) \mathrm{e}^{-i \varepsilon_{\alpha} t}|\alpha\rangle_{a s}^{0}\right] \Rightarrow 0 \\
& \lim _{t \rightarrow-\infty}\left[|\varphi(t)\rangle-\int_{\beta \alpha} \mathscr{S}_{\beta \alpha}^{*} d_{-}(\beta) \mathrm{e}^{-i \varepsilon_{s} t}|\alpha\rangle_{a s}^{0}\right] \Rightarrow 0
\end{aligned}
$$

The proof of these relations is exactly the same as the proof of (1.8), since $a$ ) and $b$ ) are the only premisses. (2.5a) is the expression of property $c$ ), which is hereby proved. It also follows as before that $\mathscr{S}$ is unitary.

$\mathscr{P}$ is not the $S$-matrix which gives the relation between the free particle states at $t=-\infty$ and $t=+\infty$. The latter is defined as follows. Let the asymptotic behaviour of $|\varphi(t)\rangle(2.4)$ be given by

$$
\begin{aligned}
& |\varphi(t)\rangle \Rightarrow \int_{\alpha} \tilde{b}(\alpha) \mathrm{e}^{-i \varepsilon_{1} t}|\alpha\rangle_{a s}^{-} \text {for } t \rightarrow+\infty \\
& |\varphi(t)\rangle \Rightarrow \int_{\alpha} b(\alpha) \mathrm{e}^{-i \varepsilon_{a} t}|\alpha\rangle_{\text {as }}^{+} \text {for } t \rightarrow-\infty
\end{aligned}
$$

where

$$
\begin{aligned}
& \left|\boldsymbol{k}_{1} a\right\rangle \text { as }=\left|\boldsymbol{k}_{1}\right\rangle|a\rangle \\
& \left|\boldsymbol{k}_{1} \mu\right\rangle_{\text {as }}^{\stackrel{ \pm}{ \pm}}=\left|\boldsymbol{k}_{1}\right\rangle|\mu\rangle_{\text {土 }}
\end{aligned}
$$

The S-matrix is then defined by

$$
\tilde{b}(\alpha)=\int_{\beta} S_{\alpha \beta} b(\beta) \text {. }
$$

To find the relation between $S$ and $\mathscr{S}$ we first define two matrices $A_{\beta \alpha}( \pm)$ by

$$
\lim _{t \rightarrow=\infty} \int_{\alpha} d(\alpha) \mathrm{e}^{-i \varepsilon_{a} t}\left[|\alpha\rangle_{a s}^{ \pm}-\int_{\beta} A_{\beta x}(+)|\beta\rangle_{a s}^{0}\right] \Rightarrow 0
$$

for arbitrary, but square integrable $d(\alpha)$. Because the states $|\alpha\rangle_{\text {as }}$ are also linearly independent they characterize linearly independent cigenstates $|\alpha\rangle_{+ \pm}$and $|\alpha\rangle_{- \pm}$(analogous to (2.2)). Since the convergence in (2.6) is strong it follows that

and

$$
|\alpha\rangle_{+ \pm}=\int_{\beta} A_{\beta \alpha}(+)|\beta\rangle_{0 \pm}
$$

$$
|\alpha\rangle- \pm=\int_{\beta} A_{\beta \alpha}(-)|\beta\rangle_{0 \pm}
$$

Because the $A( \pm)$ transform one complete orthonormal set into another they are unitary transformations. Moreover there matrixelements $A_{\alpha \beta}( \pm)$ are different from zero only if $\varepsilon_{x}=\varepsilon_{\beta}$. Using the unitarity of $A$ (土) formula (2.6) can be written as

$$
\lim _{t \rightarrow \mp \infty} \int_{\alpha} d(\alpha) \mathrm{e}^{-i \delta \alpha t}\left[|\alpha\rangle_{\text {as }}^{0}-\int_{\beta} A_{\alpha \beta}^{*}( \pm)|\beta\rangle_{\text {as }}^{ \pm}\right] \Rightarrow 0
$$


With (2.7) and (2.5) the asymptotic behaviour of $|\varphi(t)\rangle(2.4)$ can be calculated. We find

$$
b(\alpha)=\int_{\beta} d_{+}(\beta) A_{\beta \alpha}^{\star}(+)
$$

and

$$
\tilde{b}(\alpha)=\int_{\beta \gamma} A_{\gamma \alpha}^{*}(-) \mathscr{S}_{\gamma \beta} d_{+}(\beta)
$$

so that

$$
S_{\alpha \beta}=\int_{\gamma \delta} A_{\gamma \delta}^{*}(-) \mathscr{S}_{\gamma \delta} A_{\delta \beta}(+) \text { or } S=A^{+}(-) \mathscr{S} A(+) .
$$

$S$ is again unitary. The operators $A( \pm)$ can be calculated as soon as the phase shifts for the two-particle scattering are known. This will be shown in the appendix for the case of particles of different types.

The factorization (2.8) of the $S$-matrix amounts to a separation of the two-particle effects from the genuine three-particle effects as described by $\mathscr{P}$. The calculation of $\mathscr{S}$ is as difficult as all other three-body problems, but the extra complications mentioned in the introduction have been removed.

The method given here answers question $\alpha$ ) of the introduction, if we assume the existence of a.s. states with the properties $a, b$ and $c$. However, we have not been able to give a general answer to question $\beta$ ). In order to do so we must construct simple a.s. states and this we can only do for the case that no more than one pair of particles can be bound. Since our method is exact it can also be used to find the range of validity of certain approximations. One such approximation will be made in the next section, where we will consider the scattering of a free particle by a composite one.

\$3. Elastic scattering. We want to calculate the scattering of a particle of the first kind by a bound state of the particles 2 and 3 . We take the total energy sufficiently small so that the bound state cannot be broken up into its constituent particles. The $S$-matrix elements of interest are therefore $S_{\alpha \beta}$ with both $|\alpha\rangle_{a s}^{ \pm}$and $|\beta\rangle_{a s}^{ \pm}$of the form

$$
\left|\boldsymbol{k}_{1} a\right\rangle_{a s}^{ \pm}=\left|\boldsymbol{k}_{1} a\right\rangle_{a s}^{0}=\left|\boldsymbol{k}_{1}\right\rangle|a\rangle .
$$

The only non-vanishing $A_{\alpha \beta}( \pm)$ which come into play are diagonal unit elements, so that $S_{\alpha \beta}=\mathscr{S}_{\alpha \beta}$. The problem is now to solve the Schrödinger equation and to find the expansion coefficients $C_{\beta \alpha}(+)$ in (2.2). The approximation we make consists of putting $C_{\beta \alpha}(+)=0$, except when both $|\alpha\rangle_{a s}^{0}$ and $|\beta\rangle_{a s}^{0}$ are of the form (3.1). Even below the threshold for break up this is not exact since intermediate states of the form $\left|\boldsymbol{k}_{1} \boldsymbol{k}_{2} \boldsymbol{k}_{\mathbf{3}}\right\rangle_{\text {as }}^{0}$ certainly play a role. The approximation neglects this effect.

Introducing a more specific notation we write the bound states as $|a\rangle=$ $=|\boldsymbol{p}, n\rangle$, where $\boldsymbol{p}$ is the total momentum and $n$ is a set of quantum numbers, 
which label the internal state. The energy of this state is $\omega_{n}(p)=\left(p^{2} / 4 m\right)-\Delta_{n}$, where $\Delta_{n}$ is the binding energy and $m$ is the mass of a free particle. In the coordinate representation the wave function of this state is

$$
\left\langle\boldsymbol{r}_{2} \boldsymbol{r}_{\mathbf{3}} \mid \boldsymbol{p}, n\right\rangle=(2 \pi)^{-\frac{\mathrm{z}}{\mathrm{z}}} \mathrm{e}^{i \boldsymbol{p} \cdot\left(\boldsymbol{r}_{2}+\boldsymbol{r}_{3}\right) / 2} g_{n}\left(\boldsymbol{r}_{2}-\boldsymbol{r}_{\mathbf{3}}\right)
$$

with the normalization

$$
\int \mathrm{d} \boldsymbol{r} g_{m}^{*}(\boldsymbol{r}) g_{n}(\boldsymbol{r})=\delta_{m n} .
$$

In the elastic approximation we try to find an eigenstate of $H$ of the form $\left|\boldsymbol{k}, \boldsymbol{p}_{0} n_{0}\right\rangle^{+}=\left|\boldsymbol{k}, \boldsymbol{p}_{0} n_{0}\right\rangle_{a s}-\sum_{n} \int_{\boldsymbol{k}, \boldsymbol{p}} \frac{C(\boldsymbol{k}, \boldsymbol{p} n) \delta\left(\boldsymbol{k}+\boldsymbol{p}-\boldsymbol{k}_{0}-\boldsymbol{p}_{\mathbf{0}}\right)}{\omega_{n}(p)+\varepsilon(k)-\omega_{n_{\mathbf{0}}}\left(p_{0}\right)-\varepsilon\left(k_{0}\right)-i 0}|\boldsymbol{k}, \boldsymbol{p} n\rangle_{a s}$

The Schrödinger equation becomes $C\left(\boldsymbol{k}^{\prime}, \boldsymbol{p}^{\prime} n^{\prime}\right)=$

$$
=W\left(\boldsymbol{k}^{\prime} \boldsymbol{p}^{\prime} n^{\prime} \mid \boldsymbol{k}_{\mathbf{0}} \boldsymbol{p}_{0} n_{\mathbf{0}}\right)-\sum_{n} \int_{\boldsymbol{k}, \mathbf{p}} \frac{C(\boldsymbol{k}, \boldsymbol{p} n) W\left(\boldsymbol{k}^{\prime} \boldsymbol{p}^{\prime} n^{\prime} \mid \boldsymbol{k} \boldsymbol{p} n\right) \delta\left(\boldsymbol{k}+\boldsymbol{p}-\boldsymbol{k}_{\mathbf{0}}-\boldsymbol{p}_{0}\right)}{\omega_{n}(p)+\varepsilon(k)-\omega_{n_{\mathbf{0}}}\left(p_{0}\right)-\varepsilon\left(k_{0}\right)-i 0},
$$

with

and

$$
\boldsymbol{k}^{\prime}+\boldsymbol{p}^{\prime}=\boldsymbol{k}_{0}+\boldsymbol{p}_{0}
$$

$$
\begin{aligned}
W\left(\boldsymbol{k}^{\prime} \boldsymbol{p}^{\prime} n^{\prime} \mid \boldsymbol{k} \boldsymbol{p} n\right) \delta\left(\boldsymbol{k}^{\prime}+\boldsymbol{p}^{\prime}-\boldsymbol{k}-\boldsymbol{p}\right)= \\
={ }_{a s}\left\langle\boldsymbol{k}^{\prime}, \boldsymbol{p}^{\prime} n^{\prime}|\boldsymbol{V}| \boldsymbol{k}, \boldsymbol{p} n\right\rangle_{a s}-\left\langle\boldsymbol{p}^{\prime} n^{\prime}|V| \boldsymbol{p} n\right\rangle \delta\left(\boldsymbol{k}^{\prime}-\boldsymbol{k}\right) .
\end{aligned}
$$

Defining $\hat{v}_{i j}(\boldsymbol{k})$ by

we find

$$
\hat{\nu}_{i j}(\boldsymbol{k})=(2 \pi)^{-3} \int \mathrm{d} \boldsymbol{r} \mathrm{e}^{i \boldsymbol{k} \cdot \boldsymbol{r}} v_{i j}(\boldsymbol{r})
$$

$W\left(\boldsymbol{k}^{\prime} \boldsymbol{p}^{\prime} n^{\prime} \mid \boldsymbol{k} \boldsymbol{p} n\right)=\hat{v}_{12}\left(\boldsymbol{k}-\boldsymbol{k}^{\prime}\right) F_{n^{\prime} n}\left(\frac{\boldsymbol{k}-\boldsymbol{k}^{\prime}}{2}\right)+\hat{v}_{31}\left(\boldsymbol{k}^{\prime}-\boldsymbol{k}\right) F_{n^{\prime} n}\left(\frac{\boldsymbol{k}^{\prime}-\boldsymbol{k}}{2}\right)$,

where the "formfactors" of the bound states are

$$
F_{n^{\prime} n}(\boldsymbol{k})=\int \mathrm{d} \boldsymbol{r} \mathrm{e}^{i \boldsymbol{k} \cdot \boldsymbol{r}} g_{n^{\prime}}^{\star}(\boldsymbol{r}) g_{n}(\boldsymbol{r}) .
$$

The form of the integral equation (3.2) shows that the elastic approximation anounts to the introduction of an effective interaction $W$ between the free and the composite particle, with matrixelements given by (3.3) and (3.4). This interpretation is not correct when two or three particles are identical. The states $|\boldsymbol{k}, \boldsymbol{p} n\rangle_{\boldsymbol{a}}$ are not orthogonal in that case and (3.2) takes a somewhat more complicated form.

Equation (3.2) - or its modification when exchange effects are present can be used for further investigations into the problem of elastic scattering of a particle by a composite particle.

Acknowledgement. It is a pleasure to acknowledge some discussions with Professor N. G. van Kampen. 


\section{APPENDIX.}

The non-trivial part of $A_{\alpha \beta}( \pm)$ is given by $A_{\mu \nu}( \pm)$, where

$$
|\mu\rangle_{ \pm}=\int_{\nu} A_{v \mu}( \pm)|v\rangle_{0}
$$

and $|\mu\rangle_{ \pm}$and $|\mu\rangle_{0}$ are defined in (1.1) and represent two-particle scattering states. In the representation in which the operators for the total momentum, the kinetic energy, the total angular momentum and its $z$-component are diagonal, the states $|\mu\rangle_{0}$ can be written as

$$
\left|\boldsymbol{p}_{0}, \boldsymbol{k}_{0} l_{0} m_{0}\right\rangle_{0}=N\left(k_{0} l_{0}\right)\left|\boldsymbol{p}_{0}, k_{0} l_{0} m_{0}\right\rangle-\int_{k} \frac{m d\left(k \mid k_{0}\right)}{\left(k^{2}-k_{0}^{2}\right)_{P}}\left|\boldsymbol{p}_{0}, k l_{0} m_{0}\right\rangle .
$$

An alternative form is

$$
\begin{aligned}
& \left|\boldsymbol{p}_{0}, k_{0} l_{0} m_{0}\right\rangle_{0}= \\
& =\left\{N\left(k_{0}, l_{0}\right) \mp \frac{i \pi m d\left(k_{0} \mid k_{0}\right)}{2 k_{0}}\right\}\left|\boldsymbol{p}_{0}, k_{0} l_{0} m_{0}\right\rangle-\int_{k} \frac{m d\left(k \mid k_{0}\right)}{\left(k^{2}-k_{0}^{2} \pm \mathrm{i} 0\right)}\left|\boldsymbol{p}_{0}, k l_{0} m_{0}\right\rangle
\end{aligned}
$$

The expression between brackets is of unit magnitude since the eigenstates are normalized. If we take $N\left(k_{0}, l_{0}\right)$ and $d\left(k_{0} \mid k_{0}\right)$ real it can therefore be written as $\mathrm{e}^{ \pm i \varphi_{1}\left(k_{0}\right)}$. From this the two-particle $S$-matrix is obtained. It is given by

$$
S\left(\boldsymbol{p}, k l m \mid \boldsymbol{p}^{\prime}, k^{\prime} l^{\prime} m^{\prime}\right)=\delta\left(\boldsymbol{p}-\boldsymbol{p}^{\prime}\right) \delta\left(k-k^{\prime}\right) \delta_{l l^{\prime}} \delta_{m m^{\prime}} \mathrm{e}^{2 i q_{l}(k)},
$$

so that $\varphi_{l}(k)=\delta_{l}(k)$, where $\delta_{l}(k)$ are the usual phase shifts. Comparing the asymptotic behaviour of the states (A.1) and (A.2) the $A_{\mu v}( \pm)$ are immediately seen to be given by

$$
A_{ \pm}\left(\boldsymbol{p}, k l m \mid \boldsymbol{p}^{\prime}, k^{\prime} l^{\prime} m^{\prime}\right)=\delta\left(\boldsymbol{p}-\boldsymbol{p}^{\prime}\right) \delta\left(k-k^{\prime}\right) \delta_{l l} \delta_{m m^{\prime}} \mathrm{e}^{ \pm i \delta_{l}(l k)}
$$

The $A( \pm)$ are indeed unitary and on the energy shell.

Received $19 \cdot 9-61$

\section{REFERENCES}

1) Van Hove, L., Physica 21 (1955) 901, 22 (1956) 343.

2) See e.g. Møller, C., Dan. mat. fys. medd. 23 (1945) no. 1.

3) Brenig, W. and II aag, R., Fortschritte der Physik 7 (1959) 183

4) Jauch, J. M., Helvetica Physica Acta 31 (1958) 127 and 661.

5) Lane, A. M. and Thomas, R. G., Rev, mod. Phys. a0 (1958) 257.

6) Van Ka mpen, N. G., Ned. T. Natuurk. 24 (1958) 29.

7). Nuclear Forces and the Few-Nucleon Problem, Pergamon Press, Oxford, 1960, vol. I and II; Mott, N. F. and Massey, H. S. W., The Theory of Atomic Collisions, 2nd edition, Oxford Univ. Press;

Glauber, R. J., Phys. Rev. 100 (1955) 242;

Fulton, T. and Schwed, Ph., Phys. Rev. 115 (1959) 973;

Massey, H. S. W., Progress in Nuclear Physics, Pergamon Press, London, 1953, vol. 3, p. 235.

8) Ekstein, H., Phys. Rev. 101 (1956) 880, Nuovo Cim. (1956) 1017. 